\title{
Energy-Efficient Protocol for Multi-hop Sensor Networks
}

\author{
Ahmad Al Masri*, Gilles Y. Delisle \\ Electrical and Computer Engineering Department, Laval University, Quebec City, Canada. \\ * Corresponding author. Email: ahmad.almasri.1@ulaval.ca \\ Manuscript submitted December 8, 2014; accepted April 4, 2015. \\ doi: 10.17706/ijcee.2015.7.3.189-198
}

\begin{abstract}
Among the main problems which remains to be addressed in wireless sensor networks (WSNs), the energy-efficiency and the lifetime of these networks, particularly those that are deployed over large areas, are two of the most important one. The hierarchical routing protocols are playing a key role in reducing energy consumption in WSN and, for most of these protocols, the cluster-head (CH) is also used as a relay node to transmit data to the base station. This is inefficient since they consume more energy than the other nodes of the networks. This paper reports on a new algorithm, named multi-hop energy-efficient (MHEE), which maximizes the lifetime of the network. It is based on a reliable selection mechanism for the relay nodes between the cluster heads $(\mathrm{CH})$ and the base station in order to share evenly the energy consumption amongst the nodes of the networks. The results obtained show that the proposed protocol exceeds the performance existing representatives WSN routing protocols.
\end{abstract}

Key words: Wireless sensor networks, multi-hop, routing protocol, energy efficient, clustering, network lifetime.

\section{Introduction}

A wireless sensor networks (WSNs) is comprised of spatially distributed sensor nodes, where each one of them contains units for sensing, processing, and communicating data packet. This is done with many other nodes acting as relays before reaching final destination and the wireless transmission uses more energy than data processing. In general, each sensor node is both a sensor and a router, and its computing ability, storage capacity, communication ability and power supply are limited. Typically, these nodes report sensed data to a base station for further processing. They are equipped with low-cost small-capacity batteries which are, in most cases, non-rechargeable and irreplaceable. Therefore, the energy efficiency plays an important in the WSN to extend the network lifetime [1], [2].

Many routing protocols have been proposed to reduce the energy consumption between nodes for efficient data transmission, but their implementation in large network is still a problem because they do not achieve the expected lifetime required by the industrial applications [3]. Thus, each routing protocol has specific features depending on the application and the network architecture to improve the optimum energy efficiency; these characteristics are being designed using localization and clustering of the data, a well-known technique for increasing network lifetime, improve energy efficiency and equilibrate energy consumption amongst the nodes [4].

The clustering protocols are mainly considered as cross-layering techniques for designing energy efficient hierarchical wireless sensor networks. The basic principle of the protocol is to organize the sensor nodes into groups called clusters. In each cluster, a node is selected as the cluster-head. Instead of sending data directly to the base station, each node sends data to their corresponding $\mathrm{CH}$. Once the $\mathrm{CH}$ has received 
the data of all nodes in clusters, it realizes the fusion and compression of data and communicates with the base station via single or multi-hop [5].

The communication model that WSN uses is either single-hop or multi-hop [6]-[9]. Since energy consumption in wireless systems is directly proportional of the square of the distance, single-hop communication is expensive in terms of energy consumption. Most of the routing algorithms use multi-hop communication model since it is more energy efficient in terms of energy consumption. However, with multi hop communication causes energy unbalancing, because the nodes in proximity of the base station play the role of relay node for data transmission which causes depleting the energy quickly. This greatly affect the network lifetime.

\section{Current Knowledge}

Different methods for clustering the data routing are available in the public literature and a very brief review of these as well as a useful comparison to establish their respective potential are given below:

\subsection{Low Energy Adaptive Clustering (LEACH)}

This protocol is one of the first hierarchical routing protocols used in WSN to increase the network lifetime. In LEACH, the nodes organize themselves into local cluster; each cluster is formed of $\mathrm{CH}$ node and another nodes. The $\mathrm{CH}$ can be selected randomly and then rotate this role to evenly distribute the energy load among the sensors in the network [7], [8].

The operation of LEACH is divided into rounds, each round having two phases; a set-up phase when the clusters are organized and a steady-state phase when the nodes are transmitting the data to their respective $\mathrm{CH}$ which, in turn, transmit the whole set of compressed data to the base station.

During the set-up phase, each sensor node choose a random number between 0 and 1 . If this number is below the threshold $T(n)$ as indicated by eq.(1), the node will broadcast itself as being the $\mathrm{CH}$. The non- $\mathrm{CH}$ nodes choose the $\mathrm{CH}$ with greater signal strength and join the cluster.

$$
T(n)= \begin{cases}\frac{P}{1-P \times\left(r \bmod \frac{1}{P}\right)} & \text { if } n \in G \\ 0 & \text { if not }\end{cases}
$$

where $G$ is the set of nodes that did not become CHs in the last $N / k$ rounds, $k$ is the number of expected CHs, $N$ is the total number of nodes, $P$ is the percentage of the number of CHs is selected equal to $k / N$ and $r$ is the current number of round.

In the steady-state phase, data are transferred from the non-CHs nodes to the $\mathrm{CH}$ and on to the base station. After a certain period of time, set-up phase will start again.

\subsection{Two Level LEACH (TL-LEACH)}

The TL-LEACH [8] is an extension of LEACH protocol. In this protocol, the cluster-heads can be decomposed in two-level, primary and secondary. The secondary CH's collects data from the primary $\mathrm{CH}^{\prime} \mathrm{s}$ member, and relays the data to the base station. The structure of two levels reduces the number of nodes when data is transmitted from source to the base station and reducing total energy consumption.

\subsection{Energy Efficient Clustering Hierarchy (EECH)}

EECH protocol [8] uses an energy based threshold calculation to elect CHs in the network, in which there is a higher probability for a node with more energy to be selected as $\mathrm{CH}$ than a node with less energy. It uses 
multi-hop communication between a $\mathrm{CH}$ and the BS to reduce energy consumption in the network.

\subsection{Multi-hop Routing LEACH (MR-LEACH)}

In the MR-LEACH [9], the network divided in optimal number of layer, the BS is involved in the selection of the $\mathrm{CH}$ and it publishes a Time Division Multiple Access (TDMA) schedule for each CHs layer. Based on this schedule, each CHs layer collaborates with the adjacent CHs layers to transmit sensor's data to the BS with multi-hop routing.

\subsection{Improved LEACH (ILEACH)}

The ILEACH protocol [10] uses the residual energy of the nodes to select the $\mathrm{CH}$, preventing the nodes with weak energy to become $\mathrm{CH}$. An energy management function to equilibrate the energy consumption between the $\mathrm{CHs}$ has been proposed and a data aggregation tree is designed to send data $\mathrm{CH}$ to the BS in multi-hop mode.

Table 1 summarizes the advantages and disadvantages of these different protocols.

Table 1. Comparison of Routing Protocols

\begin{tabular}{lll}
\hline \hline Protocol & Advantages & Disadvantages \\
\hline LEACH & Reduce the total data transmitted to the base station & $\begin{array}{l}\text { Cannot be applied in networks deployed in } \\
\text { large regions. }\end{array}$ \\
TL LEACH & $\begin{array}{l}\text { The number of nodes transmitting to the base station is } \\
\text { reduced therefore diminish the total energy consumption } \\
\text { Energy consumption is well distributed on all nodes for } \\
\text { EECH }\end{array}$ & $\begin{array}{l}\text { Similarly, this does not apply for networks } \\
\text { deployed over large areas. }\end{array}$ \\
Small networks. & $\begin{array}{l}\text { For large networks, uneven distribution of } \\
\text { nodes energy. }\end{array}$ \\
ILEACH & $\begin{array}{l}\text { Decomposition of nodes network into multi-levels. } \\
\text { Diminution of the communication distance between the }\end{array}$ & $\begin{array}{l}\text { Centralized routing protocol. } \\
\text { Fost energy consumption for the nodes closer } \\
\text { to the BS. }\end{array}$ \\
\hline \hline
\end{tabular}

\section{Network and Energy Consumption Model Used in Our Study}

A sensor field consisting of $\mathrm{N}$ sensors deployed randomly in a rectangular space is considered. Our node network model has the following properties:

- The base station is located far away from the node network.

- All nodes are equally capable of becoming CHs.

- All the nodes are homogeneous and each node is assigned with a distinctive identifier (ID).

- Sensor nodes are aware of their location or position in the network.

- All nodes are capable of sending data to the base station.

- The base station and the nodes are stationary after deployment.

For the protocol analysis, a simple model (Fig. 1) of communication energy dissipation is used where the transmitter and the receiver are using energy to power the electronic devices and the power amplifier.

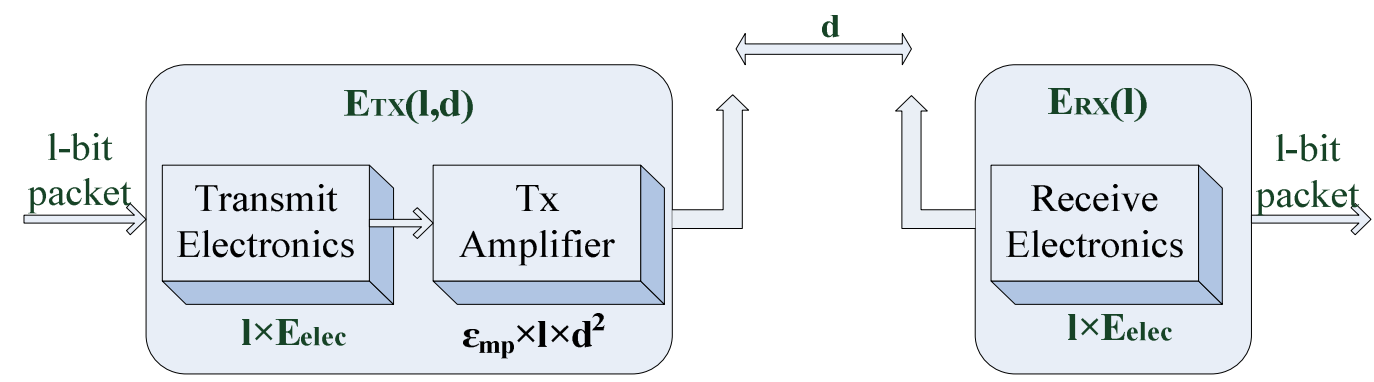

Fig. 1. Simple energy dissipation model. 
Depending upon the distance between the transmitter and the receiver, a typical energy consumption model is adopted, both free space and multipath fading channel models are proportional to $d^{2}$ and in $d^{4}$ power loss are used. If the distance is below a threshold $d_{0}$, the free space $(f s)$ model is used; on the contrary, multipath propagation model is used. Therefore, to transmit $l$-bit packet over a distance $d$, the required energy is given by the following equations [7]:

$$
\begin{gathered}
E_{T X}(l, d)= \begin{cases}l E_{\text {elec }}+l \varepsilon_{f s} d^{2} & \text { if } d<d_{0} \\
l E_{\text {elec }}+l \varepsilon_{m p} d^{4} & \text { if } d \geq d_{0}\end{cases} \\
d_{0}=\sqrt{\frac{\varepsilon_{f s}}{\varepsilon_{m p}}}
\end{gathered}
$$

where $E_{\text {elec }}$ is the energy consumption per bit in the transmitter and receiver circuitry [7], $d_{0}$ is the maximum distance for which the transmission factors change values, $\varepsilon_{f s}$ is the free space model's amplifier energy consumption, and $\varepsilon_{m p}$ is the multiple attenuation model amplifier energy consumption .

The energy consumed to receive $l$-bit data is represented in (3):

$$
E_{R X}(l, d)=l E_{\text {elec }}
$$

\section{The Proposed Protocol}

In the clustering protocol, the $\mathrm{CH}$ is receiving all the nodes data and merges them in a single packet before sending it to the base station. The energy consumption in each cluster is proportional to the surface of the cluster since in large size clusters the distance between the $\mathrm{CH}$ and the member nodes increases.

After all the data have been received, the $\mathrm{CH}$ transmits the packet to the base station from a relay node to diminish the separation distance between the $\mathrm{CH}$ and the base station. For the large surfaces, two mechanisms are available; in the first mechanism, the clusters number are increase therefore increasing the inter-cluster energy consumption and ,in the second mechanism, the number of clusters is diminished, yielding a diminution of the inter-cluster energy consumption and an increase of intra-cluster energy consumption.

In the clustering protocols briefly covered in Section 2, the CHs closer to the base station are also playing the role of nodes at the same time and this is not optimal for two reasons. First, when the base station is far from the node network, it is preferable to diminish the transmission distance by choosing closer nodes such as relay nodes. When this role is attributed to the CHs such as in these protocols, this leads to the rapid depletion of their energy and it is more difficult to use them as relay nodes, leading to a shorter operational lifetime for the network. The second reason is that when a node is transmitting and receiving at the same time, it consumes of course more energy than if it just transmits or receives. Therefore, when two tasks are attributed to the same nodes, particularly closer to the end of the lifetime of the network, could lead to total energy exhaustion.

Using these considerations, it can therefore be concluded that there are two main problems to be dealt with, namely the cluster surface and the relay nodes. To address these issues, our new approach proposes to devise the network surface in two or more levels and elect a relay node for each of these levels. It differs from most of the known protocols that are based on the distance to the base station and the nodes energy level. Our objective is to have uniform energy consumption between all the nodes of the network. 
Our protocol, named MHEE (multi-hop energy efficient) is divided into three phases, A) the identification and clustering, B) the relay nodes election and C) the data routing.

\subsection{Identification and Clustering}

This phase has two steps, namely the identification of the nodes as a function of the levels and the CHs election and the nodes clustering.

\subsubsection{Identification}

The networks dedicated to the coverage of large surfaces lose more energy when transmitting data between the CHs and the base station. To diminish the energy consummation between the network nodes, it is divided into two or more levels. Fig. 2 shows the result for two levels (1\&2).

When the distribution of the nodes in the surveillance zone is accomplished, each node has the information concerning its coordinates $(x i, y i)$, as well as the length and the width of the total zone. If the ordinate $y i$ of node $i$ is larger than half the length of the zone, then node $i$ is at level 2, the level closer to the base station. Conversely, if it is smaller, then node $i$ belongs to level 1 , the more remote level from the base station.

\subsubsection{Clustering}

When each level has been determined, the CHs election is done using the $T(n)$ level concept of eq. (1) of Section 2. Then each elected $\mathrm{CH}$ broadcasts an advertisement message to the rest of the nodes that belongs to the same level to invite them to joint their clusters and each one will join the closer $\mathrm{CH}$. Thereafter, each CH creates a TDMA schedule for each node in its cluster. This procedure is conceptually pictured in Fig. 2.

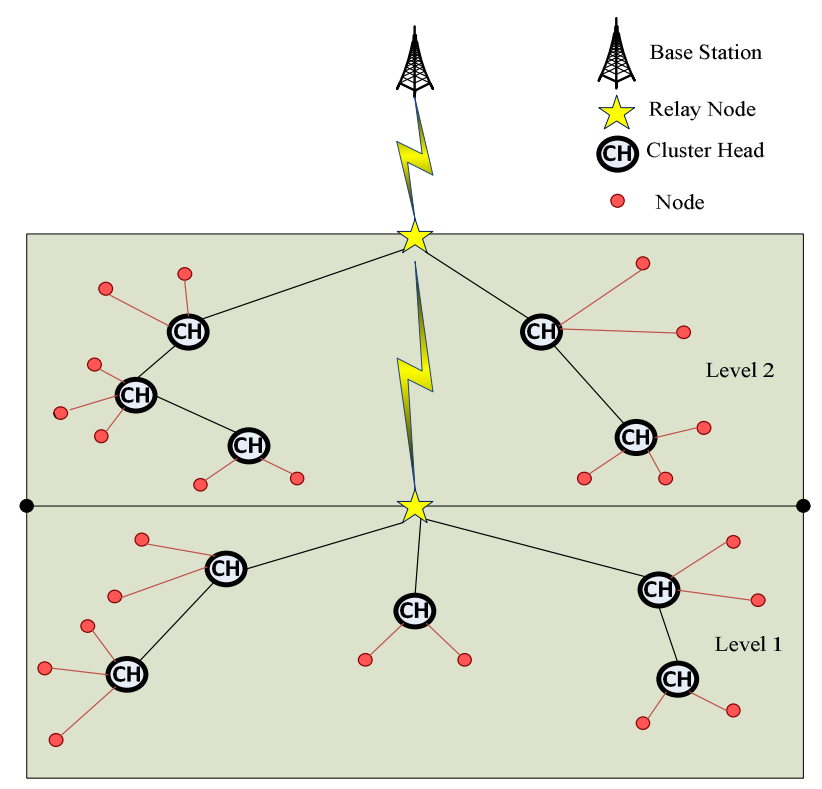

Fig. 2. MHEE protocol.

\subsection{Relay Nodes Election}

The distribution of energy between the nodes of the network permits to extend the network lifetime. For an equitable share of energy consumption between the network nodes, a relay node is elected for each level and its characteristics are: a maximum energy level and a minimum distance with the base station. This node receives all the data from the same level CHs and, after, $N_{r 1}$ (the relay node of level 1) send the data to relay node of level 2 . Once all the data has been received by $N_{r 2}$, it transmits it directly to the base station.

The election of a relay node at level 1 is based on threshold $\mathrm{TH}_{1}$, as shown in eq. (4) below. For each level, 
each $\mathrm{CH}$ knows the position of all the other $\mathrm{CHs}$. The closer $\mathrm{CH}$ from the base station will broadcast a message to all the nodes in order to know their $\mathrm{TH}_{1}$ and their identification. With the reception of all node thresholds, the node with the larger $\mathrm{TH}_{1}$ will be elected by the $\mathrm{CH}$ as the level 1 relay node and will broadcast an advertisement message containing the identifier of $N_{r 1}$ to all the CHs of that level. This is expressed in eq. (4)

$$
\mathrm{TH}_{i 1}=\frac{E_{i}}{E_{0}}+\frac{d_{\max 1}}{d_{i_{-} \mathrm{To}_{-} \mathrm{BS}}}
$$

With $\mathrm{TH}_{i 1}$ representing the threshold of the node $i$ for level $1, E_{i}$ the residual energy of node $i, E_{0}$ the initial energy of the node, $d_{\max 1}$ the maximum distance between the surveillance zone of level 1 (as expressed in eq. 5 ) and the base station and $d_{i_{-} \text {To_BS }}$ the distance between node $i$ and the base station. This is depicted in Fig. 3.

$$
d_{\max 1}=\sqrt{\left(x_{\mathrm{BS}}\right)^{2}+\left(y_{\mathrm{BS}}\right)^{2}}
$$

The same phenomenon will occur at level 2 and will be expressed in eq. (6).

$$
\mathrm{TH}_{i 2}=\frac{E_{i}}{E_{0}}+\frac{d_{\max 2}}{d_{i_{-} \mathrm{To} \_\mathrm{BS}}}
$$

where $\mathrm{TH}_{i 2}$ representing the threshold of the node $i$ for level $2, d_{\max 2}$ is the maximum distance between the surveillance zone of level 2 and the base station (eq. 7).

$$
d_{\max 2}=\sqrt{\left(x_{\mathrm{BS}}\right)^{2}+\left(\left(y_{\mathrm{BS}}\right)-\left(\frac{y_{\text {surface }}}{2}\right)\right)^{2}}
$$

where $y_{\text {surface }}$ is the width of the node network surface.

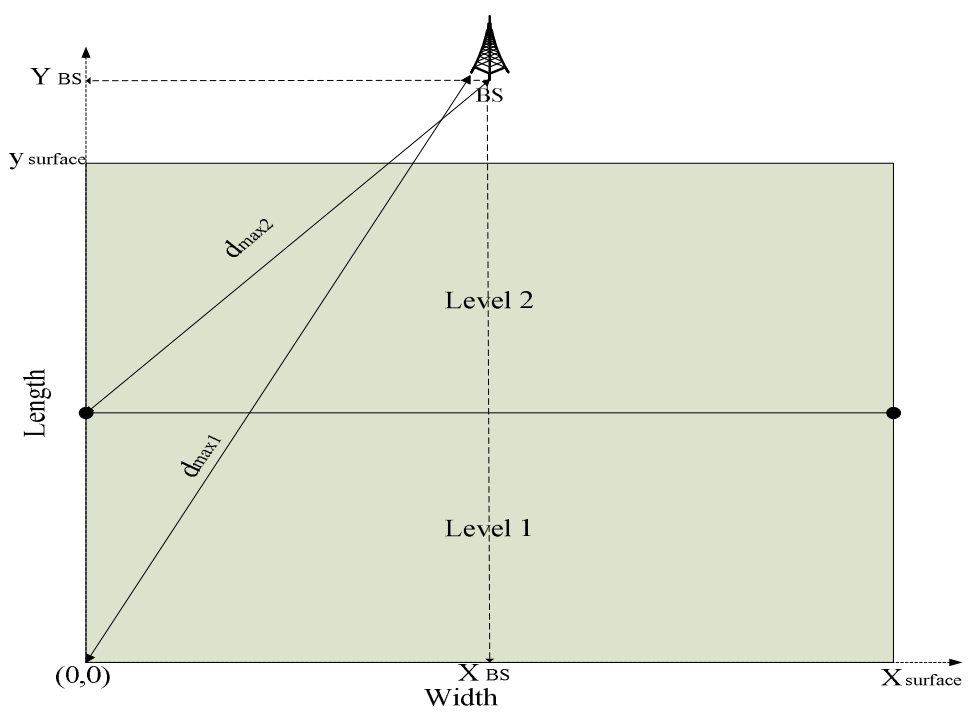

Fig. 3. Network surface for MHEE protocol. 


\subsection{Data Routing}

When the clusters are configured, the CHs are elected and the relay nodes of each level are spotted, the data routing phase to the base station begins.

In this paper, a multi-hop data communication algorithm between the CHs is propose in order to minimize the distance between $\mathrm{CH}$ and the energy consumption during transmission in each level where a $\mathrm{CH}$ will communicate with the closer $\mathrm{CH}$ in order to send the data to the corresponding relay node.

\section{Simulation Results}

Before experimentation in a real system, various clustering protocols scenarios were simulated using MATLAB. The values of the parameters used in the simulation are given in Table 2.

Table 2. Parameters Used in Simulation

\begin{tabular}{ll}
\hline \hline Parameter & Value \\
\hline Area of network & $(300 \mathrm{~m}, 300 \mathrm{~m})$ \\
Number of nodes & 300 \\
Situation of base station & $(150 \mathrm{~m}, 400 \mathrm{~m})$ \\
Packet Size & $4000 \mathrm{bits}$ \\
Eo: Initial Energy of Sensor Nodes & $1 \mathrm{~J}$ \\
do: Threshold distance & $87 \mathrm{~m}$ \\
Efs: Transmit amplifier free-space & $10 \mathrm{pJ} / \mathrm{bit} / \mathrm{m} 2$ \\
Eamp: Transmit amplifier for two-way & $0.0013 \mathrm{pJ} / \mathrm{bit} / \mathrm{m} 4$ \\
Eelec: Transceiver idle state energy consumption & $50 \mathrm{~nJ} / \mathrm{bit}$ \\
EDA: Consume Energy Data Aggregation & $5 \mathrm{~nJ} / \mathrm{bit}$ \\
Popt: Probability of optimum CH & $10 \%$ \\
\hline \hline
\end{tabular}

Fig. 4 illustrates the comparison between LEACH, ILEACH, MR-LEACH protocol and MHEE protocol in term of the lifetime per round. It clearly shows that our proposed protocol MHEE is more stable than the others since the degeneration begins later than the other algorithms.

While comparing our algorithm with the other ones, it has been noticed that the base station, when using our MHEE protocol, received information from the surveillance zone over a longer period of time.

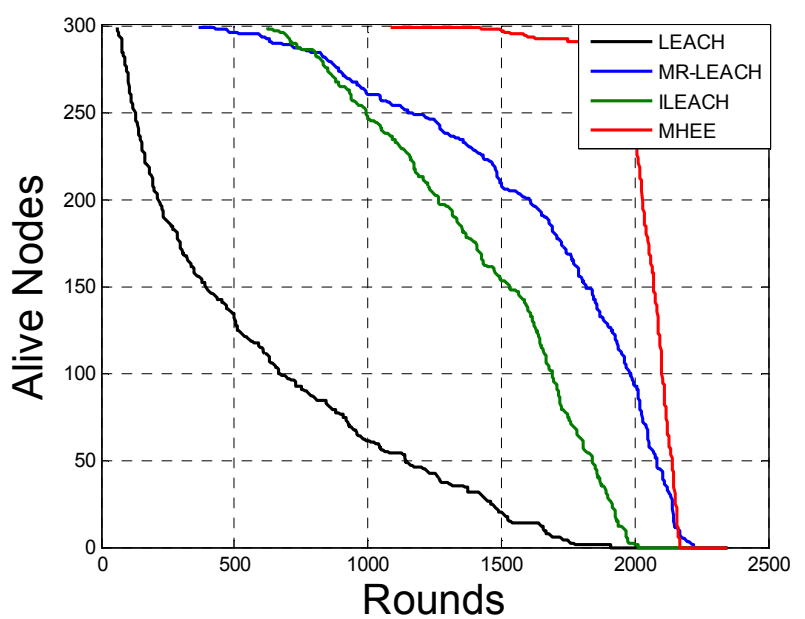

Fig. 4. Network lifetime comparison amongst LEACH, ILEACH, MR-LEACH and MHEE.

The amount of data received by each algorithm considered is shown in Fig. 5 and this result shows an increase in the amount of data received by the base station using the MHEE protocol of 74,4\%, $29 \%$ and $19,4 \%$ in regard to LEACH, ILEACH and MR-LEACH respectively. 


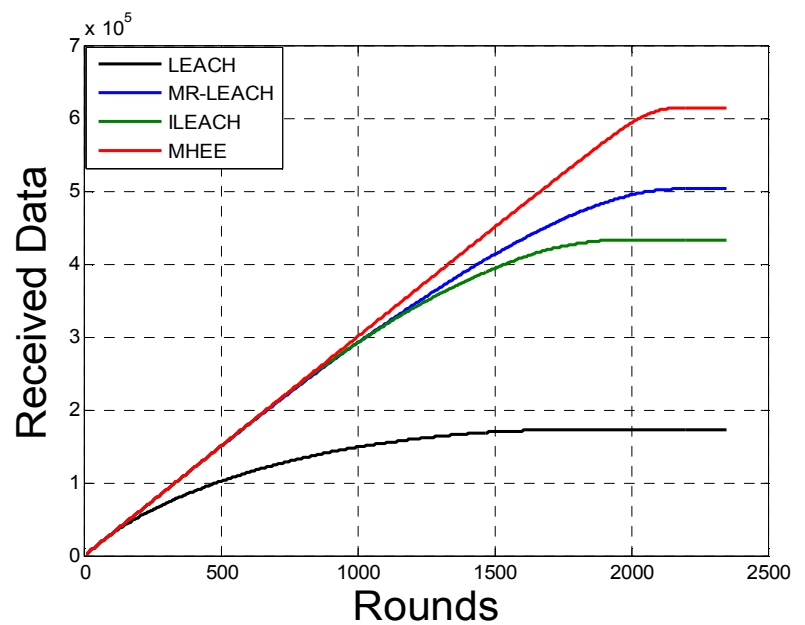

Fig. 5. The data received by the base station by round.

Fig. 6 depicts the remaining energy in the whole network for each round. This result shows clearly that the distribution of energy in the MHEE protocol driven network is above the others while being almost linear the full number of rounds.

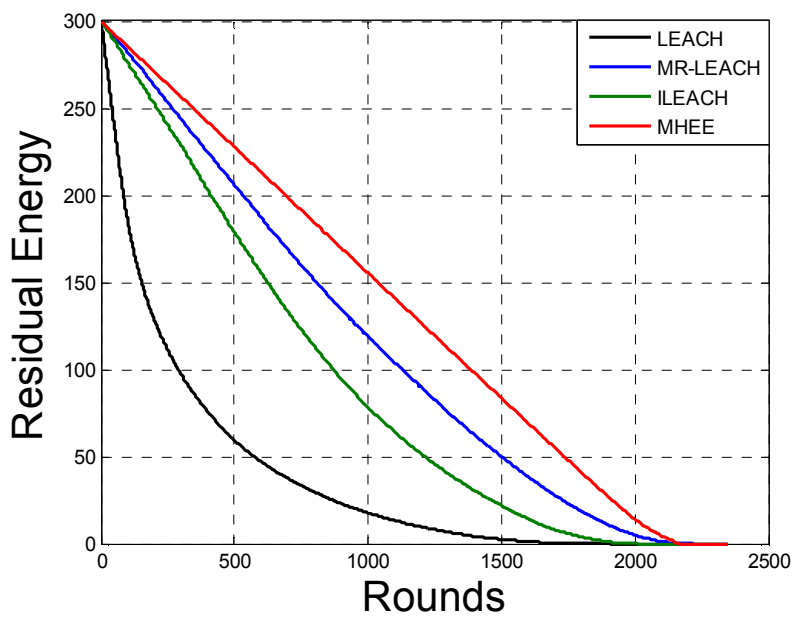

Fig. 6. Energy consumption of the WSN for each round.

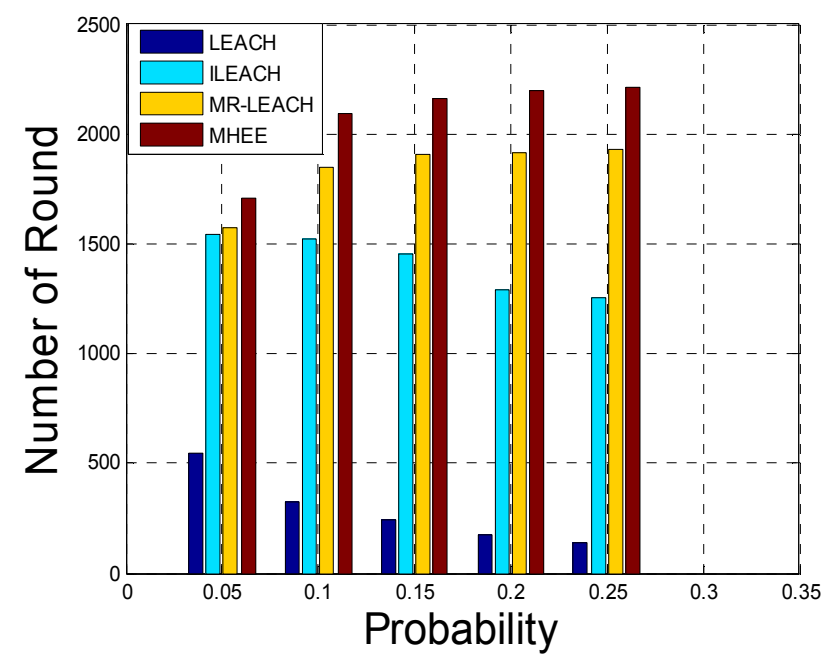

Fig. 7. The HDN for different probability of $\mathrm{CH}$ in the network. 
Fig. 7 is another result showing comparative advantages for our MHEE protocol. In this case, the probability of HDN (half dead nodes) is illustrated for different numbers of CHs in the network. For two protocols, MHEE and MR-LEACH, the more the probability of nodes is increased, the more the lifetime of HDN are increased.

Many other results will be presented and discuss at the conference.

\section{Conclusion}

This paper is proposing a novel clustering algorithm specially designed for large coverage network. The MHEE protocol divides the nodes network in two or more levels and, at each of these levels, a relay node is selected. The usefulness of this node is to distribute the energy consumption between nodes in order to increase the amount of received information. Subsequently, a multi-hop communication between the CHs is used to transmit the data to the relay node and the base station.

The proposed protocol permits to reduce the inter-cluster communication and consequently to diminish the energy consumption. Simulation results show an increase of the data received by the base station and a better distribution of the energy consumption compared to other existing protocols such LEACH, ILEACH and MR-LEACH.

\section{References}

[1] Dargie, W., \& Poellabaur, C. (2010). Fundamentals of Wireless Sensor Networks: Theory and Practice. New York, NY, USA: Wiley.

[2] Jiong, J., Sridharan, A., Krishnamachari, B., \& Palaniswami, M. (September 2010). Handling inelastic traffic in wireless sensor networks. IEEE Journal on Selected Areas in Communications, 28(7), 1105-1115.

[3] Pantazis, N. A., Nikolidakis, S. A., \& Vergados, D. D. (2013). Energy-efficient routing protocols in wireless Sensor networks: A survey. IEEE Communications Surveys \& Tutorials, 15(2), 551-591.

[4] Zhang, H. B., \& Shen, H. (2010). Energy-efficient beaconless geographic routing in wireless sensor networks. IEEE Transactions on Parallel and Distributed Systems, 21(6), 881-896.

[5] Karkvandi, H. R., Pecht, E., \& Yadid-Pecht, O. (2011). Effective lifetime-aware routing in wireless sensor networks. IEEE Sensors Journal, 11(12), 3359-3367.

[6] Stecklina, O., Langendoerfer, P., \& Goltz, C. (2013). A fair energy trade multi-hop routing in wireless sensor networks. Proceedings of 6th Joint IFIP on Wireless and Mobile Networking Conference (pp. 1-8). Dubai, UAE.

[7] Heinzelman, W. B., Chandrakasan, A. P., \& Balakrishnan, H. (2002). An application-specific protocol architecture for wireless microsensor networks. IEEE Transactions on Wireless Communications, 1(4), 660-670.

[8] Ray, A., \& De, D. (March 2012). Energy efficient cluster head selection in wireless sensor network. Proceedings of 1st International Conference on Recent Advances in Information Technology (pp. 306-311).

[9] Farooq, M. O., Dogar, A. B., \& Shah, G. A. (2010). MR-LEACH: Multi-hop routing with low energy adaptive clustering hierarchy. Proceedings of Fourth International Conference on Sensor Technologies and Applications (pp. 262-268).

[10] Yang, J., Li, Z. T., \& Lin, Y. (May 2013). An improved routing algorithm based on LEACH for wireless sensor networks. Proceedings of 25th Chinese on Control and Decision Conference (pp. 3716-3720). Guiyang, China. 


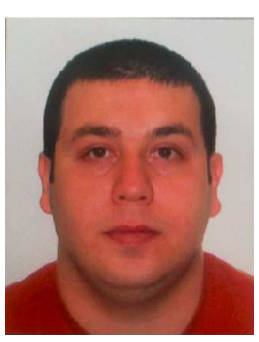

Ahmad Al Masri received license and master degrees in electronics from the Faculty of Sciences, Lebanese University in 2008 and 2009 respectively. Also he received the master of engineering degree in telecommunication from Quebec University in Chicoutimi, Canada, in 2013. Currently he is a Ph.D. candidate in electrical engineering at Laval University, Quebec, Canada. His current research interests include energy-efficient of the wireless sensor networks.

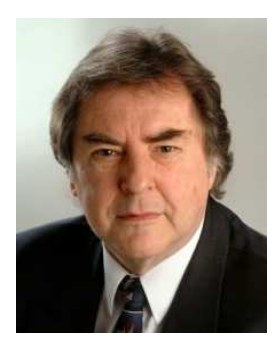

Gilles-Y. Delisle is a professor at UQAT (Université du Québec en Abitibi-Témiscamingue) since 2010 and the president of GDE Inc., which is a consulting organization in telecommunications. He is also an emeritus professor at Laval University and an adjunct professor at other four universities. He was the director of the Technology Integration Centre at Technopôle Defence and Security in Valcartier, Québec, Canada from 2008 to 2010. From June 2004 to March 2008, he was the vice-president researcher at the International Institute of Telecommunications in Montréal, Canada. Previously, he was the director and a professor at the School of Information Technology and Engineering at the University of Ottawa from 2002 to 2004 and he has been a professor of electrical and computer engineering at Laval University, Québec, Canada from 1973 to 2001, where he was the head of the department from 1977 to 1983. From June 1992 to June 1997, he was also the director of INRS-Telecommunications, a research institute which is a part of the Université du Québec. He is involved in research work in underground mines communication networks, intelligent antenna array, radar cross-section measurements and analytical predictions, mobile radio-.channel propagation modeling, personal communications and industrial realization of telecommunications equipment.

Dr. Delisle is a member of the Order of Engineers of the Province of Québec and Professional Engineers of Ontario, a fellow of Engineer's Canada, the past-president of the Canadian Engineering Accreditation Board, a fellow of the Canadian Academy of Engineering, the past Canadian president of URSI, the past president of ACFAS, a life fellow of the Institute of Electrical and Electronics Engineers (IEEE), a fellow of the Canadian Engineering Institute, the Canadian Academy of Engineering and of the Institution of Engineering and Technology (IET-UK). In 1986, he was awarded the J. Armand Bombardier prize of ACFAS for outstanding technical innovation and his work in technology transfer has been recognized by a Canada Award of Excellence in 1987. The Canadian Council of Professional Engineers has recognized its contribution to the profession by awarding him the prestigious "Meritorious Service Award for Professional Service" in 2004 and the title of fellow of Engineers Canada in 2011. Dr. Delisle has supervised the work of over 140 graduate and post-graduate students over the last 40 years. He has been elected to the Canadian Hall of Fame in telecommunications in October 2007. 